\title{
Total Synthesis of an Immunogenic Trehalose Phospholipid from Salmonella Typhi and Elucidation of Its sn-Regiochemistry by Mass Spectrometry
}

Vivek K. Mishra, ${ }^{\dagger}$ Jeffrey Buter, ${ }^{\dagger}$ Molly S. Blevins, ${ }^{\dagger}$ Martin D. Witte, ${ }^{\dagger}{ }^{\dagger}$ Ildiko Van Rhijn,, ,॥ D. Branch Moody, " Jennifer S. Brodbelt, ${ }^{\ddagger}$ and Adriaan J. Minnaard*, ${ }^{\dagger} \uparrow$

${ }^{\dagger}$ Stratingh Institute for Chemistry, University of Groningen, Nijenborgh 7, 9747 AG Groningen, The Netherlands

${ }^{\ddagger}$ Department of Chemistry, University of Texas, Austin, Texas 78712, United States

${ }^{\S}$ Department of Infectious Diseases and Immunology, School of Veterinary Medicine, Utrecht University, 3584 CL Utrecht, The Netherlands

"Department of Rheumatology, Immunology, and Allergy, Brigham and Women's Hospital, Harvard Medical School, Boston, Massachusetts 02115, United States

Supporting Information

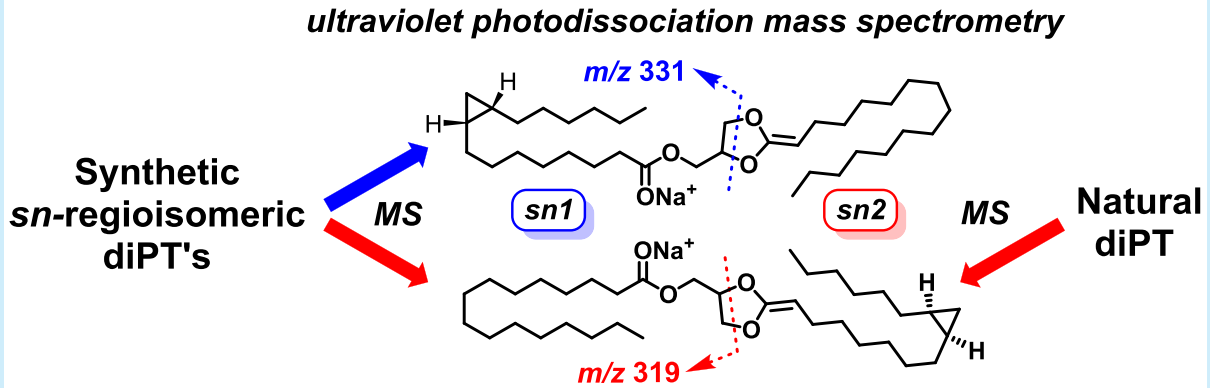

ABSTRACT: Diphosphatidyltrehalose (diPT) is an immunogenic glycolipid, recently isolated from Salmonella Typhi. Despite rigorous structure elucidation, the sn-position of the acyl chains on the glycerol backbone had not been unequivocally established. A stereoselective synthesis of diPT and its regioisomer is reported herein. Using a hybrid $\mathrm{MS}^{3}$ approach combining collisional dissociation and ultraviolet photodissociation mass spectrometry for analysis of the regioisomers and natural diPT, the regiochemistry of the acyl chains of this abundant immunostimulatory glycolipid was established.

Tipids function as the key structural components of cellular membranes and have specific biological roles in regulating cell response. ${ }^{1}$ For example, many lipids of bacterial origin trigger immune responses by binding innate receptors of the immune system. Perhaps the most studied molecule of bacterial pathogens is lipopolysaccharide (LPS), ${ }^{2}$ a virulence factor ${ }^{3}$ embedded in the outer cell membrane of Gram-negative bacteria. ${ }^{4}$ A major constituent of LPS is lipid A (2, Figure $1),{ }^{5-7}$ which acts as a potent stimulant $(\mathrm{pg} / \mathrm{mL})^{8}$ that triggers cytokine release by ligating to Toll-like receptor 4 (TLR4). ${ }^{9}$ Another important lipid that stimulates the immune system is trehalose dimycolate (TDM, 4), which is also known as the cord factor and is abundantly present in the cell wall of Mycobacterium tuberculosis. TDM binds to the macrophage-inducible $\mathrm{Ca}^{2+}$ dependent C-type lectin ${ }^{10}$ (Mincle) receptor, ${ }^{11,12}$ a carbohydrate binding protein, leading to macrophage activation and cytokine release. Lipid A and TDM thus stimulate the innate immune response. Based on detailed analysis of the aspects of the structure needed for immune response, both natural molecules have served as important scaffolds for adjuvant design ("immune response booster") in vaccine development, including lipid A and trehalose dibehenate. ${ }^{13,14}$

Recently, the first members of a new class of lipids were discovered, which consist of trehalose attached to a phosphatidic acid: phosphatidyltrehalose (PT, 3) and diphosphatidyltrehalose (diPT, 1). These glycolipids were isolated from the typhoid fever-causing, Gram-negative bacterium Salmonella Typhi. ${ }^{15} \mathrm{PT}$ and diPT bear notable structural resemblance to both TDM and LPS (Figure 1). The presence of phosphate moieties makes PT and $\mathrm{diPT}$ somewhat more closely resemble lipid A, whereas trehalose and a cyclopropyl-containing alkyl chain are congruent with TDM. The strong structural analogies of these newly discovered molecules with TDM led to immunological studies, which showed that diPT is similarly potent as a Mincle ligand and thus can serve as a new candidate for adjuvant development. ${ }^{15}$

The overall structure of PT and diPT (Figure 1) was elucidated using collisional mass spectrometry and NMR

Received: May 16, 2019

Published: June 20, 2019 


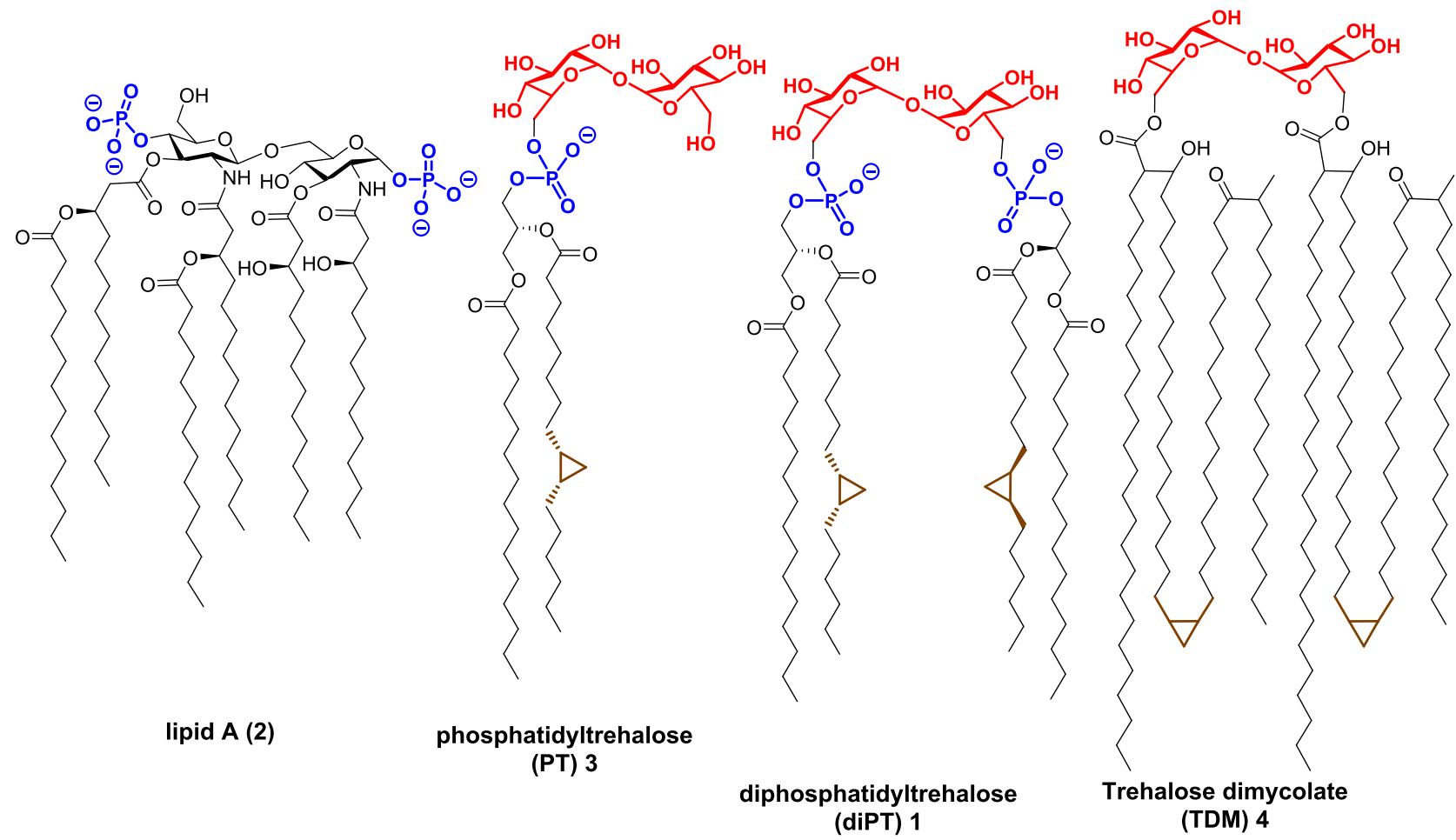

Figure 1. Structure of lipid A, phosphatidyltrehalose, diphosphatidyltrehalose, and trehalose dimycolate.

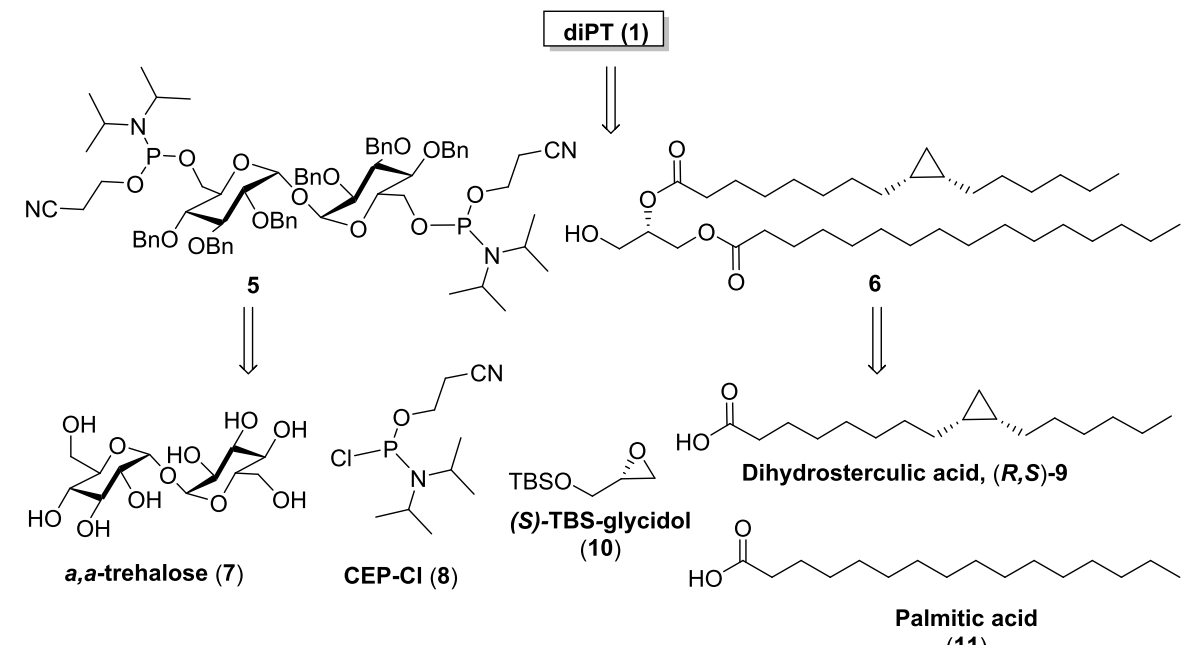

(11)

Figure 2. DiPT retrosynthesis.

spectroscopy. Two features remained unknown or uncertain, however. Whereas it is reasonable to assume that the stereochemistry of the phosphatidic acid matches the known stereochemistry of bacterial sn-glycerol-3-phosphate ${ }^{16}$ and that of the cyclopropyl function is $(9 R, 10 S)$ as found in Escherichia coli, $^{17}$ a species closely related to Salmonella, neither have been formally established. ${ }^{17,18}$ In addition, the regiochemistry of the acyl chains is unknown. A second rationale for determining the complete structure of phosphatidyl trehalose is the desire to study these compounds in immunological assays, which requires synthetic material that is entirely free from immunologically active impurities. Third, large amounts of compound are needed for human in vitro and animal in vivo studies of glycolipid function. Therefore, we undertook the total synthesis of diPT. From the outset, it was clear though that several regioisomers had to be prepared to establish the structure of the natural compound. The synthesis described herein is the first one of this new class of compounds and sets the stage for a new adjuvant design based on this novel molecular scaffold.

DiPT features two identical phosphatidic acid residues anchored to the C6-positions of trehalose. Although $C_{2^{-}}$ symmetric, synthesis by dimerization was not desired as the glycosidic bond in trehalose is particularly difficult to construct. ${ }^{19}$ In addition, trehalose itself is readily available and was therefore chosen as the starting material (Figure 2).

We explored both a phosphate and a phosphoramidite coupling strategy. The first strategy was used in the synthesis of $\beta$-mannosylphosphomycoketide, ${ }^{20}$ an antigen of Mycobacterium tuberculosis, and synthesis of acceptors ${ }^{21}$ for glycosyl transferases. The phosphoramidite coupling ${ }^{22,23}$ is an approach used 
extensively in phosphorylated protein and nucleotide synthesis. ${ }^{24-26}$

For the regio- and stereoselective synthesis of the diacylglycerol, we planned to capitalize on our earlier work in which we developed a catalytic method of epoxide ring opening that provides flexibility in the introduction of the fatty acid residues, minimizing acyl shift. ${ }^{27}$ Given the unknown regiochemistry of the acyl residues, we planned the synthesis of both regioisomers.

In our first strategy, we attempted to prepare the phosphatidic acid of $\mathbf{6}$ to activate and couple it to trehalose. Indeed, glycidol, upon reaction with dibenzyl phosphorochloridate, produced the phosphorylated glycidol (74\%), but epoxide ring opening under Jacobsen $^{28}$ and basic $(\mathrm{KOH})$ conditions with palmitic acid did not lead to product formation. On the other hand, when diglyceride 6 was treated with 2-cyanoethyl $N, N$-diisopropylchlorophosphoramidite (CEP-Cl, 8) in basic medium (Hünig's base), an inseparable mixture of regioisomers $(s n-3 / s n-2=4 / 1)$ was formed in moderate yield (46\%) due to extensive acyl shift. We discontinued this approach and decided to place the phosphoramidite units on the trehalose building block, which therefore needed a suitable protecting group. We briefly explored persilylated (TMS) ${ }_{6}$-trehalose ${ }^{29}$ that was treated with $\mathrm{CEP}-\mathrm{Cl}$ to produce the corresponding persilylated bisphosphoramidite. However, this compound was unstable under the required phosphoramidite coupling conditions. As an alternative approach, we decided to make perbenzyl ether 12 , according to a literature procedure, ${ }^{30}$ which was subsequently treated with CEP-Cl (8) to give bisphosphoramidite $\mathbf{5}$ in good yield (Scheme $1)$.

Scheme 1. Bisphosphoramidite 5 Synthesis

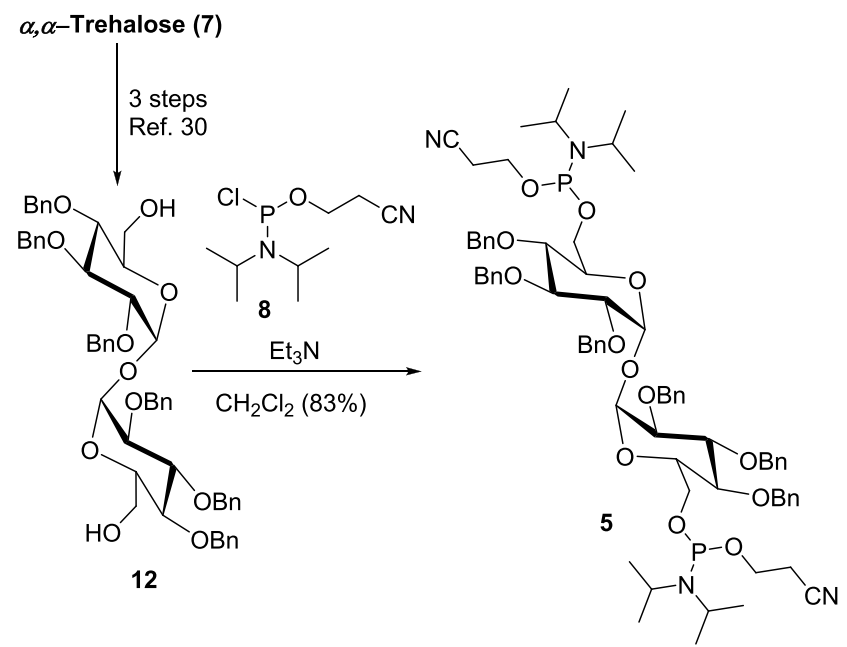

Next, the synthesis of diglyceride 6 commenced with the preparation of the cis-cyclopropyl fatty acid. A versatile method developed by Williams et al., based on rhodium-catalyzed cyclopropenation, ${ }^{31}$ was used with some modifications to prepare 9 and its enantiomer. In short, intermediate 14, obtained from octyne in four steps (Scheme 2), was subjected to oxidation with Dess-Martin periodinane to produce aldehyde 15, which was further used in a Wittig reaction to give alkene 16 in $86 \%$ yield $(Z / E=8 / 2) .{ }^{32,33}$ Reduction of 16 to the desired fatty acid $(R, S)-9(87 \%)$ was effected by diimide, in situ generated from $\mathrm{H}_{2} \mathrm{~N}-\mathrm{NH}_{2} \cdot \mathrm{H}_{2} \mathrm{O}$ and a flavin catalyst reported earlier. ${ }^{34}$ Transition-metal-catalyzed hydrogenation is
Scheme 2. Synthesis of Diacylglycerol 6

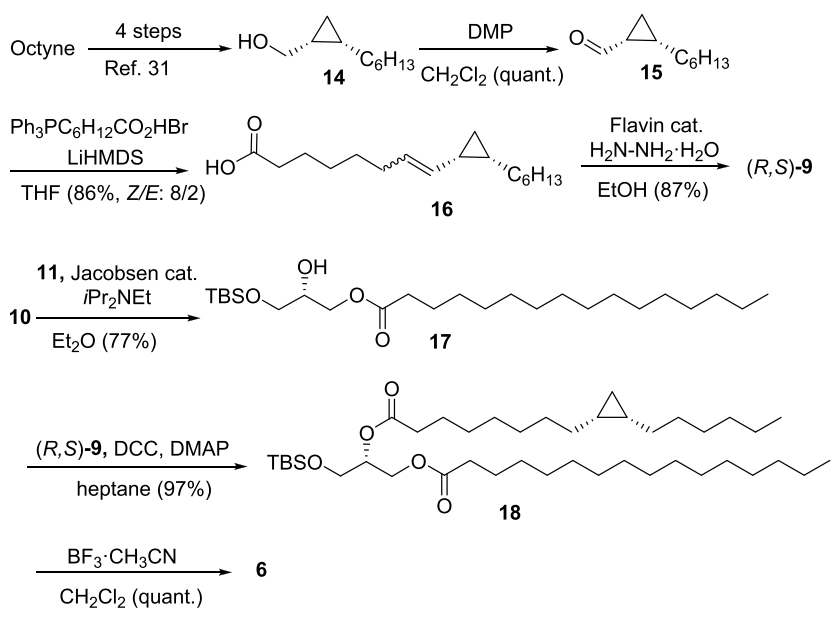

not suitable here as it leads to ring opening of the cyclopropane. The analytical data of $(R, S)-9$ and its enantiomer were in agreement with that of previously reported synthetic dihydrosterculic acid. ${ }^{31,35}$

Next, silyl-protected glycidol 10 (Scheme 2) was subjected to epoxide ring opening by employing Jacobsen's catalyst with palmitic acid 11 to give acyl glycerol $17 .^{28}$ Subsequently, 17 was reacted with $(R, S)-9$ under Steglich conditions to produce protected diglyceride 18. Diacylglycerols are prone to acyl migration, ${ }^{36}$ but as previously reported, $\mathrm{BF}_{3} \cdot \mathrm{CH}_{3} \mathrm{CN}$-mediated deprotection of $\mathbf{1 8}$ under carefully controlled conditions produced advanced intermediate $\mathbf{6}$, without NMR-detectable migration.

With all building blocks in hand, a careful study of the phosphoramidite coupling was performed using dipalmitoyl glycerol, leading to straight-chain $\operatorname{diPT}_{4}$ (see Supporting Information) and trehalose bisphosphoramidite 5. The progress of the reaction was monitored by proton-decoupled ${ }^{31} \mathrm{P}$ NMR. Initially, instead of a phosphite intermediate, we observed acyl migration when solid dicyanoimidazole (DCI) was used as the catalyst for the reaction. Surprisingly, using a DCI solution ( $1 \mathrm{M}$, in $\mathrm{CH}_{3} \mathrm{CN}$ ), no NMR-detectable acyl migration was observed. Complete consumption of starting material was observed within $1 \mathrm{~h}$, and the produced intermediate was oxidized in situ into the corresponding phosphate 24 with $t \mathrm{BuOOH}$. We found that an excess of $t \mathrm{BuOOH}$ led to oxidation of the benzyl ethers into the corresponding benzoyl esters, as confirmed by HRMS. The issue was solved using just a slight excess of $t \mathrm{BuOOH}$ (2.5 equiv) at 0 ${ }^{\circ} \mathrm{C}$. The optimized conditions were employed successfully to couple 5 and 6 to get phosphate 19 in an acceptable yield (67\%, Scheme 3). The final deprotections were delicate, and we observed a clean elimination of the cyanoethyl groups in 19 by employing DBU for $5 \mathrm{~min}$ to produce phosphate 20. Finally, hydrogenolysis with a combination of $\mathrm{Pd} / \mathrm{C}$ and $\mathrm{Pd}(\mathrm{OH})_{2} / \mathrm{C}$ (2:3) in a $\mathrm{CH}_{2} \mathrm{Cl}_{2} / \mathrm{MeOH} /$ water mixture produced the desired product diPT (1, called "diPT 1 " from here on) in excellent yield. Using the synthetic sequence outlined, two analogues of $\operatorname{diPT}_{1}$ (1) were synthesized in good overall yields by replacing $(R, S)-9$ with $(S, R)-9$ to provide $\operatorname{diPT}_{2}(31)$. We switched the dihydrosterculic acid position (from $s n-2$ to $s n-1$ ) in the phosphatidic acid, leading to $\operatorname{diPT}_{3}(38)$.

With $\operatorname{diPT}_{1} 1$, its stereoisomer 31 , its regioisomer 38 , and the natural isolate in hand, their ${ }^{1} \mathrm{H}$ and ${ }^{13} \mathrm{C}$ resonances could be compared (Tables S1 and S2). DiPT 1 has a very close match 
Scheme 3. DiPT 1 Synthesis End Game

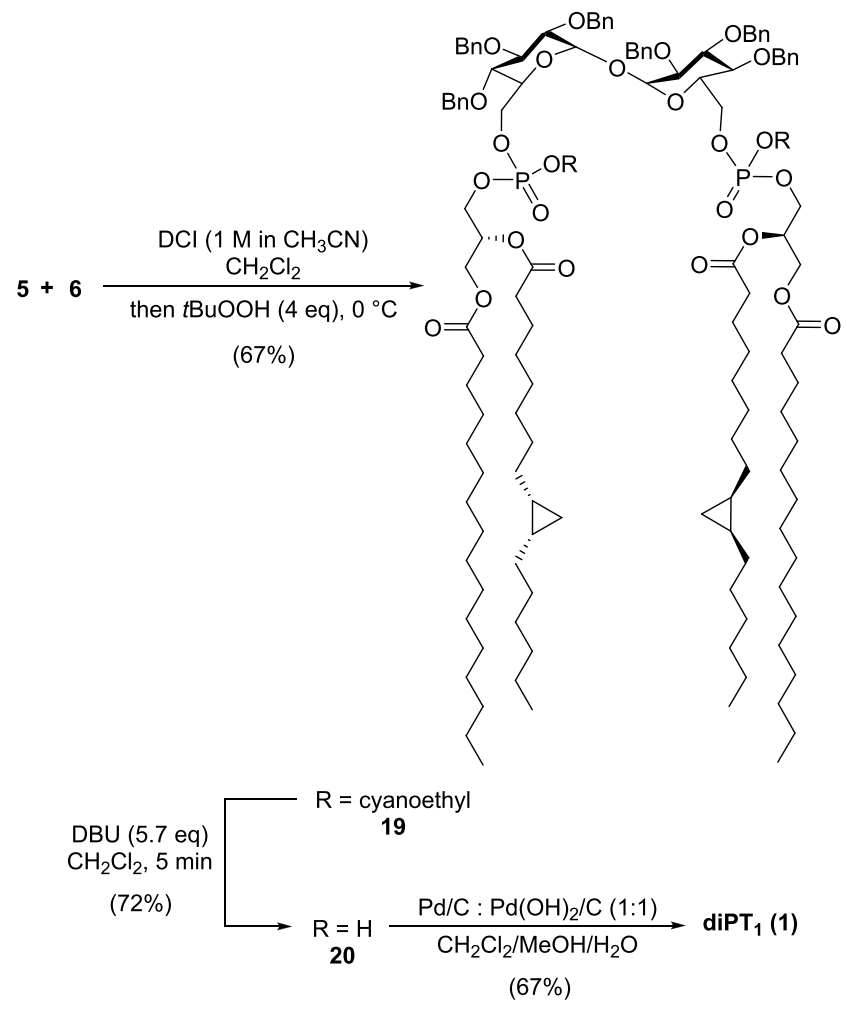

with the natural material. The anomeric protons appear as a doublet in $\mathbf{1}(\delta, 5.08 \mathrm{ppm}, J=3.8 \mathrm{~Hz})$ and natural diPT $(\delta, 5.08$ ppm, $J=3.7 \mathrm{~Hz})$, whereas these are observed as an apparent singlet in $31(\delta, 5.10 \mathrm{ppm})$ and $38(\delta, 5.06 \mathrm{ppm})$. Apart from this difference, the diastereotopic signals of the cyclopropyl group have a similar chemical shift $(\delta,-0.32 \mathrm{ppm})$ in $\mathbf{1}$ and natural diPT, which differs by $0.04 \mathrm{ppm}$ in 31 and 38 . The only minor differences are resonances appearing in $\mathbf{1}(\delta, 1.82-1.66 \mathrm{ppm})$ and in natural $\operatorname{diPT}(\delta, 2.37-2.12 \mathrm{ppm})$, which are attributed to unidentifiable impurities. The NMR data showed a strong correspondence between $\operatorname{diPT}_{1} 1$ and natural diPT. The snposition of the acyl chains on each glycerol backbone, however, could not be determined nor could the cyclopropane ring in the acyl chains be localized. To solve this structural puzzle, we resorted to ultraviolet photodissociation MS (UVPD-MS), ${ }^{37-39}$ a method that has proven useful for detailed structural characterization of complex lipids.

Unlike traditional collisional activation methods, UVPD affords direct localization of subtle structural elements in lipids including double bonds and cyclopropane rings. ${ }^{37,38,40}$ For example, UVPD allows the positions of cyclopropane rings in lipids to be pinpointed via generation of a diagnostic pair of fragment ions spaced $14 \mathrm{Da}$ apart. ${ }^{40}$ Here, this method was applied for analysis of diPT lipids in negative ion mode. Negative mode MS1 spectra for all diPT species are provided in Figure S1, allowing confirmation of chemical formula via high-resolution high-mass accuracy data. As shown in Figure 3a-d, cyclopropane localization in all three synthetic diPT species as well as the natural diPT lipid is possible via UVPD of the doubly deprotonated precursor $[\mathrm{M}-2 \mathrm{H}]^{2-}$ of $m / z 812$. Cyclopropane is determined to reside at $\mathrm{C} 9$ as counted from the acyl chain carbonyl carbon, as illustrated by the fragments in green font in the map in Figure 3e and observed as diagnostic ions of $m / z 756$ and $m / z 763$ in Figure $3 a-c$, corresponding to a difference of 14 Da.

Next, a hybrid collisional activation/UVPD-MS ${ }^{3}$ method was employed as previously described for determination of $s n$ configuration. ${ }^{37}$ In brief, sodium-adducted diPT species were fragmented using higher-energy collisional dissociation (HCD) for generation of five-membered cyclic sodium-adducted species of $\mathrm{m} / z 585$ via loss of the headgroup, followed by subsequent activation of the product by UVPD. As displayed in Figure 4a, $\operatorname{diPT}_{1} 1$ yields mainly a product of $m / z 319$ upon hybrid $\mathrm{HCD} /$

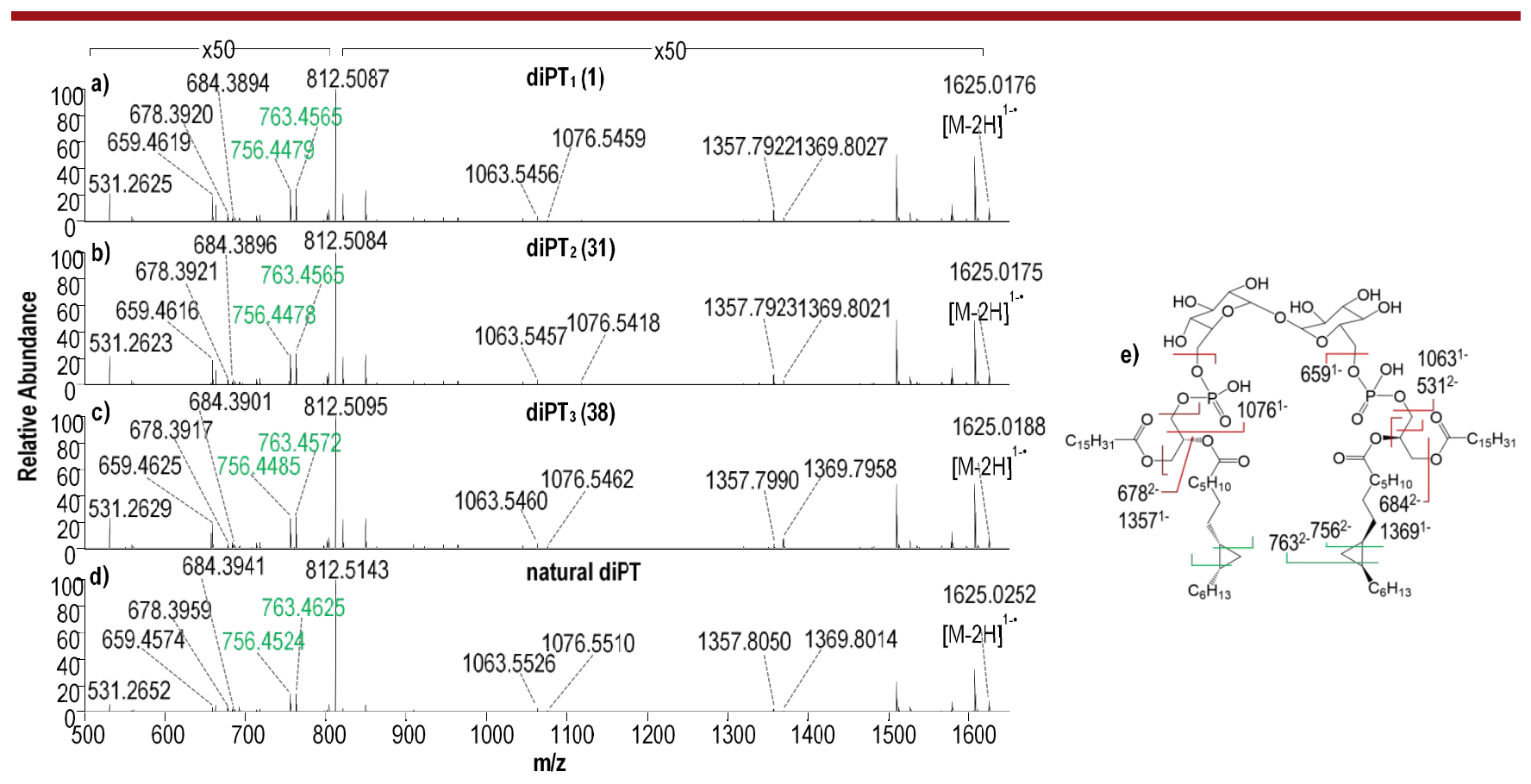

Figure 3. Negative mode UVPD mass spectra of $[\mathrm{M}-2 \mathrm{H}]^{2-}\left(m / z\right.$ 812) for $(\mathrm{a}) \mathrm{diPT}_{1},(\mathrm{~b}) \mathrm{diPT}_{2},(\mathrm{c}) \mathrm{diPT}_{3}$, and (d) natural diPT. (e) Representative fragmentation of $\mathrm{diPT}_{3}$. 


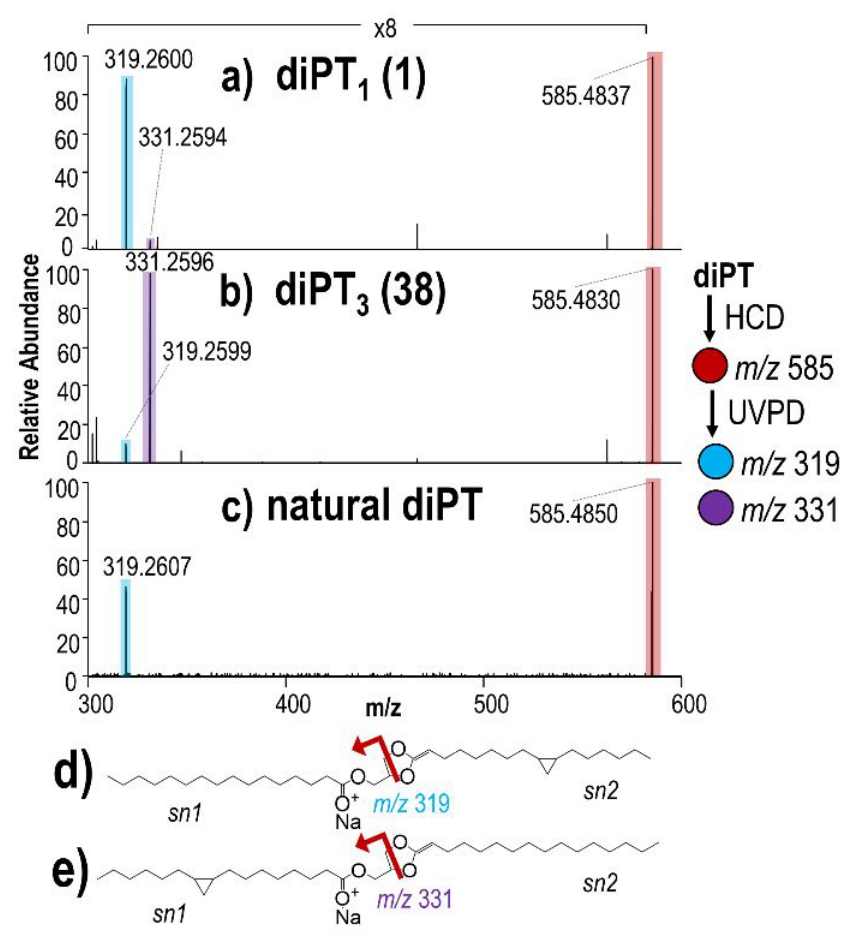

Figure 4. UVPD mass spectra of $[\mathrm{M}-2 \mathrm{H}]^{2-}\left(m / z\right.$ 812) for (a) $\operatorname{diPT}_{1}$, (b) $\mathrm{diPT}_{3}$, and (c) natural diPT in negative mode with $(\mathrm{d}, \mathrm{e})$ fragment ion maps.

UVPD activation, with a lower abundance product of $m / z 331$. These two fragment ions are attributed to photolytic cleavage across the dioxolane ring, resulting in sodium-adducted fatty acid allyl esters of each $s n-1$ fatty acid chain. Cyclic structures for the product ion of $m / z 585$ and the UVPD fragment ions of $m / z$ 319 and $m / z 331$ are shown in Figure 4d,e. In contrast to this fragmentation pattern, the ion of $m / z 585$ of $\operatorname{diPT}_{3} 38$ produces mainly an ion of $m / z 331$ upon UVPD, with a low-abundance ion of $m / z$ 319. Natural diPT exclusively produces an ion of $m / z$ 319 , suggesting that the $s n$-configuration of natural diPT aligns with the $s n$-configuration of synthetic $\operatorname{diPT}_{1}$. These results indicate that natural diPT contains the 16:0 acyl chain at the $s n-1$ fatty acid position and the 17:1(C9 $\Delta)$ acyl chain at the $s n-2$ fatty acid position. The data also suggest that both $\operatorname{diPT}_{1}$ and $\operatorname{diPT}_{3}$ contain low-abundance impurities of the opposite $s n$-isomer which were not detected using NMR analysis. These impurities are suspected to have arisen in the epoxide opening $(\mathbf{1 0}+\mathbf{1 1})$ or the DCI-mediated phosphate coupling $(5+6)$. Examples of positive mode $\mathrm{MS}^{1}$ and MS/MS spectra for diPT samples are provided in Figures S2 and S3. In summary, UVPD-MS provided characterization of key structural elements including cyclopropane localization and $s n$-determination, i.e., regiochemistry, for both synthetic and natural diPT species.

In conclusion, the synthesis of diphosphatidyltrehalose (diPT, 1), an immunogenic trehalose phospholipid from Salmonella Typhi, has been achieved. The synthesis of $\mathbf{1}\left(\mathrm{diPT}_{1}\right)$ and its regioisomer $38\left(\mathrm{diPT}_{3}\right)$ allowed the determination of the fatty acid regiochemistry in natural diPT. It is shown that UVPD-MS is able to solve this long-standing problem of fatty acid regiochemistry by comparing the hybrid $\mathrm{MS}^{3} \mathrm{HCD} / \mathrm{UVPD}$ fragmentation patterns of natural and synthetic compounds. Additionally, using this state-of-the-art technique, we determined the cyclopropyl group to be in the 9,10-position of the acyl chain as previously inferred. Access to synthetic, naturally occurring diPT, its stereoisomer 31, its regioisomer 38, and the availability of a robust synthetic route can now be used to determine a structure-activity relationship for activation of Mincle. Moreover, the synthesis allows for the design of other novel adjuvants which can greatly facilitate vaccine development.

\section{ASSOCIATED CONTENT}

\section{Supporting Information}

The Supporting Information is available free of charge on the ACS Publications website at DOI: 10.1021/acs.orglett.9b01725.

Experimental data, NMR, and MS (PDF)

\section{AUTHOR INFORMATION}

\section{Corresponding Author}

*E-mail: a.j.minnaard@rug.nl. ORCID $\odot$

Martin D. Witte: 0000-0003-4660-2974 Adriaan J. Minnaard: 0000-0002-5966-1300 Notes

The authors declare no competing financial interest.

\section{ACKNOWLEDGMENTS}

We thank P. van der Meulen, Dr. J. Kemmink, and R. Sneep (University of Groningen) for assistance on analyses. Funding from the NIH (R01 AI116604, J.S.B.) and (GM103655, M.S.B.), the Welch Foundation (F-1155, J.S.B.), and the UT System for support of the UT System Proteomics Core Facility Network is gratefully acknowledged. The Dutch Science Foundation NWO is acknowledged for funding.

\section{REFERENCES}

(1) Muro, E.; Atilla-Gokcumen, G. E.; Eggert, U. S. Lipids in cell biology: how can we understand them better? Mol. Biol. Cell 2014, 25, $1819-1823$.

(2) Raetz, C. R.; Reynolds, C. M.; Trent, M. S.; Bishop, R. E. Lipid A modification systems in gram-negative bacteria. Annu. Rev. Biochem. 2007, 76, 295-329.

(3) Raetz, C. R.; Whitfield, C. Lipopolysaccharide endotoxins. Annu. Rev. Biochem. 2002, 71, 635-700.

(4) Kong, Q.; Six, D. A.; Liu, Q.; Gu, L.; Wang, S.; Alamuri, P.; Raetz, C. R.; Curtiss, R., 3rd Phosphate groups of lipid A are essential for Salmonella enterica serovar Typhimurium virulence and affect innate and adaptive immunity. Infect. Immun. 2012, 80, 3215-24.

(5) Kabanov, D. S.; Prokhorenko, I. R. Structural analysis of lipopolysaccharides from Gram-negative bacteria. Biochemistry (Moscow) 2010, 75, 383-404.

(6) Mayer, H.; Weckesser, J. Different lipid A types in lipopolysaccharides of phototrophic and related non-phototrophic bacteria. FEMS Microbiol. Lett. 1988, 54, 143-153.

(7) Raetz, C. R.; Guan, Z.; Ingram, B. O.; Six, D. A.; Song, F.; Wang, X.; Zhao, J. Discovery of new biosynthetic pathways: the lipid A story. J. Lipid Res. 2009, 50 (Suppl), S103-S108.

(8) Mata-Haro, V.; Cekic, C.; Martin, M.; Chilton, P. M.; Casella, C. R.; Mitchell, T. C. The Vaccine Adjuvant Monophosphoryl Lipid A as a TRIF-Biased Agonist of TLR4. Science 2007, 316, 1628-1632.

(9) Vaure, C.; Liu, Y. A comparative review of toll-like receptor 4 expression and functionality in different animal species. Front. Immunol. 2014, 5, 316.

(10) (a) Dambuza, I. M.; Brown, G. D. C-type lectins in immunity: recent developments. Curr. Opin. Immunol. 2015, 32, 21-27. (b) Williams, S. J. Sensing Lipids with Mincle: Structure and Function. Front. Immunol. 2017, 8, 1662. 
(11) Ishikawa, E.; Ishikawa, T.; Morita, Y. S.; Toyonaga, K.; Yamada, H.; Takeuchi, O.; Kinoshita, T.; Akira, S.; Yoshikai, Y.; Yamasaki, S. Direct recognition of the mycobacterial glycolipid, trehalose dimycolate, by C-type lectin Mincle. J. Exp. Med. 2009, 206, 28792888.

(12) Desel, C.; Werninghaus, K.; Ritter, M.; Jozefowski, K.; Wenzel, J.; Russkamp, N.; Schleicher, U.; Christensen, D.; Wirtz, S.; Kirschning, C.; Agger, E. M.; da Costa, C. P.; Lang, R. The Mincle-activating adjuvant TDB induces MyD88-dependent Th1 and Th17 responses through IL-1R signaling. PLoS One 2013, 8, No. e53531.

(13) Holten-Andersen, L.; Doherty, T. M.; Korsholm, K. S.; Andersen, P. Combination of the Cationic Surfactant Dimethyl Dioctadecyl Ammonium Bromide and Synthetic Mycobacterial Cord Factor as an Efficient Adjuvant for Tuberculosis Subunit Vaccines. Infect. Immun. 2004, 72, 1608-1617.

(14) Decout, A.; Silva-Gomes, S.; Drocourt, D.; Barbe, S.; Andre, I.; Cueto, F. J.; Lioux, T.; Sancho, D.; Perouzel, E.; Vercellone, A.; Prandi, J.; Gilleron, M.; Tiraby, G.; Nigou, J. Rational design of adjuvants targeting the C-type lectin Mincle. Proc. Natl. Acad. Sci. U. S. A. 2017, $114,2675-2680$.

(15) Reinink, P.; Buter, J.; Mishra, V. K.; Ishikawa, E.; Cheng, T.-Y.; Willemsen, P. T. J.; Porwollik, S.; Brennan, P. J.; Heinz, E.; Mayfield, J. A.; Dougan, G.; van Els, C. A.; Cerundolo, V.; Napolitani, G.; Yamasaki, S.; Minnaard, A. J.; McClelland, M.; Moody, D. B.; Van Rhijn, I. Discovery of trehalose phospholipids reveals functional convergence with mycobacteria. J. Exp. Med. 2019, 216, 757-771.

(16) Lombard, J.; Lopez-Garcia, P.; Moreira, D. The early evolution of lipid membranes and the three domains of life. Nat. Rev. Microbiol. 2012, 10, 507-515.

(17) Hildebrand, J. G.; Law, J. H. Fatty Acid Distribution in Bacterial Phospholipids. The Specificity of the Cyclopropane Synthetase Reaction. Biochemistry 1964, 3, 1304-1308.

(18) Grogan, D. W.; Cronan, J. E., Jr. Cyclopropane ring formation in membrane lipids of bacteria. Microbiol. Mol. Biol. Rev. 1997, 61, 429441.

(19) Cumpstey, I. Intramolecular aglycon delivery. Carbohydr. Res. 2008, 343, 1553-1573.

(20) van Summeren, R. P.; Moody, D. B.; Feringa, B. L.; Minnaard, A. J. Total Synthesis of Enantiopure $\beta$-d-Mannosyl Phosphomycoketides from Mycobacterium tuberculosis. J. Am. Chem. Soc. 2006, 128, 45464547.

(21) Riley, J. G.; Xu, C.; Brockhausen, I. Synthesis of acceptor substrate analogs for the study of glycosyltransferases involved in the second step of the biosynthesis of $\mathrm{O}$-antigen repeating units. Carbohydr. Res. 2010, 345, 586-597.

(22) Vargeese, C.; Carter, J.; Yegge, J.; Krivjansky, S.; Settle, A.; Kropp, E.; Peterson, K.; Pieken, W. Efficient activation of nucleoside phosphoramidites with 4,5-dicyanoimidazole during oligonucleotide synthesis. Nucleic Acids Res. 1998, 26, 1046-1050.

(23) Roget, A.; Bazin, H.; Teoule, R. Synthesis and use of labelled nucleoside phosphoramidite building blocks bearing a reporter group: biotinyl, dinitrophenyl, pyrenyl and dansyl. Nucleic Acids Res. 1989, 17, 7643-7651.

(24) Silverberg, L. J.; Dillon, J. L.; Vemishetti, P. A simple, rapid and efficient protocol for the selective phosphorylation of phenols with dibenzyl phosphite. Tetrahedron Lett. 1996, 37, 771-774.

(25) Sinha, N. D.; Biernat, J.; McManus, J.; Köster, H. Polymer support oligonucleotide synthesis XVIII1.2): use of $\beta$-cyanoethyi-N,Ndialkylamino-/ $\mathrm{N}$-morpholino phosphoramidite of deoxynucleosides for the synthesis of DNA fragments simplifying deprotection and isolation of the final product. Nucleic Acids Res. 1984, 12, 4539-4557.

(26) Koizumi, K.; Maeda, Y.; Kano, T.; Yoshida, H.; Sakamoto, T.; Yamagishi, K.; Ueno, Y. Synthesis of 4'-C-aminoalkyl-2'-O-methyl modified RNA and their biological properties. Bioorg. Med. Chem. 2018, 26, 3521-3534.

(27) Fodran, P.; Minnaard, A. J. Catalytic synthesis of enantiopure mixed diacylglycerols - synthesis of a major M. tuberculosis phospholipid and platelet activating factor. Org. Biomol. Chem. 2013, 11, 6919-6928.
(28) Jacobsen, E. N.; Kakiuchi, F.; Konsler, R. G.; Larrow, J. F.; Tokunaga, M. Enantioselective catalytic ring opening of epoxides with carboxylic acids. Tetrahedron Lett. 1997, 38, 773-776.

(29) Johnson, D. A. Simple procedure for the preparation of trimethylsilyl ethers of carbohydrates and alcohols. Carbohydr. Res. 1992, 237, 313-318.

(30) Gilbertson, S. R.; Chang, C.-W. T. Synthesis of New Disugar Phosphine Ligands and Their Use in Asymmetric Hydrogenation. J. Org. Chem. 1995, 60, 6226-6228.

(31) Shah, S.; White, J. M.; Williams, S. J. Total syntheses of ciscyclopropane fatty acids: dihydromalvalic acid, dihydrosterculic acid, lactobacillic acid, and 9,10-methylenehexadecanoic acid. Org. Biomol. Chem. 2014, 12, 9427-9438.

(32) Coxon, G. D.; Knobl, S.; Roberts, E.; Baird, M. S.; Al Dulayymi, J. R.; Besra, G. S.; Brennan, P. J.; Minnikin, D. E. The synthesis of both enantiomers of lactobacillic acid and mycolic acid analogues. Tetrahedron Lett. 1999, 40, 6689-6692.

(33) Coxon, G. D.; Al Dulayymi, J. a. R.; Morehouse, C.; Brennan, P. J.; Besra, G. S.; Baird, M. S.; Minnikin, D. E. Synthesis and properties of methyl 5-(1'R,2'S)-(2-octadecylcycloprop-1-yl)pentanoate and other $\omega-19$ chiral cyclopropane fatty acids and esters related to mycobacterial mycolic acids. Chem. Phys. Lipids 2004, 127, 35-46.

(34) Smit, C.; Fraaije, M. W.; Minnaard, A. J. Reduction of CarbonCarbon Double Bonds Using Organocatalytically Generated Diimide. J. Org. Chem. 2008, 73, 9482-9485.

(35) Sauvageau, J.; Ryan, J.; Lagutin, K.; Sims, I. M.; Stocker, B. L.; Timmer, M. S. Isolation and structural characterisation of the major glycolipids from Lactobacillus plantarum. Carbohydr. Res. 2012, 357, $151-156$.

(36) Kodali, D. R.; Tercyak, A.; Fahey, D. A.; Small, D. M. Acyl migration in 1,2-dipalmitoyl-sn-glycerol. Chem. Phys. Lipids 1990, 52, $163-170$

(37) Williams, P. E.; Klein, D. R.; Greer, S. M.; Brodbelt, J. S. Pinpointing Double Bond and sn-Positions in Glycerophospholipids via Hybrid $193 \mathrm{~nm}$ Ultraviolet Photodissociation (UVPD) Mass Spectrometry. J. Am. Chem. Soc. 2017, 139, 15681-15690.

(38) Klein, D. R.; Brodbelt, J. S. Structural Characterization of Phosphatidylcholines Using $193 \mathrm{~nm}$ Ultraviolet Photodissociation Mass Spectrometry. Anal. Chem. 2017, 89, 1516-1522.

(39) Klein, D. R.; Holden, D. D.; Brodbelt, J. S. Shotgun Analysis of Rough-Type Lipopolysaccharides Using Ultraviolet Photodissociation Mass Spectrometry. Anal. Chem. 2016, 88, 1044-1051.

(40) Blevins, M.; Klein, D.; Brodbelt, J. S. Localization of Cyclopropane Modifications in Bacterial Lipids via $213 \mathrm{~nm}$ Ultraviolet Photodissociation Mass Spectrometry. Anal. Chem. 2019, 91, 68206828. 